\title{
Paper
}

\section{Predicting Long Lifetimes Using Accelerated Reliability Techniques}

\author{
Ray GIBSON
}

HID Lamp Development Lab, Philips Lighting, 7265 Route 54, Bath, New York, USA

Received March 29, 2011, Accepted July 27, 2011

\begin{abstract}
A continual challenge in lamp development is the need to rapidly test products that have lifetime ratings in the tens of thousands of hours. Traditional life testing, under controlled conditions, remains a mainstay test procedure. However, highly reliable statistical analysis methods allow lamp developers to estimate long lifetimes at a fraction of the testing time. These techniques usually involve the well known Weibull distribution together with historic data, multi-level accelerated stress testing, and virtual failure analysis to reduce the necessary testing times for establishing product life and reliability.
\end{abstract}

KEYWORDS: reliability, HID lighting, ceramic metal halide, accelerated test, Weibull distribution, Bayes analysis

\section{Introduction}

In lighting, the lifetime of a source is traditionally stated as the average $50 \%$ (median) failure point of a population of lamps. With the advancement of ceramic discharge metal halide lamps (CDM), commercial life times have reached 30,000 hours ${ }^{1}$. Under normal 11 hours on, one hour off cycle testing that allows 8000 hours of test time per year, it would take three or more years to reach the half life point of these lamps. When introducing new products, this extreme time frame is unworkable. In consequence to these long lifetimes, evaluation of life performance raises several questions that must be considered early in the test phase:

1. What is the product life, if there are no failures in the testing?

2. How many products need to be tested?

3. How can accelerated testing predict life?

4. What other performance metrics can be predicted other than product failure?

The answers to these questions can be found by using established reliability techniques and analysis that are common in other industries, but not as common in lighting, and especially in HID lighting. In this paper it is shown how these techniques are used for projecting lamp life using historic data together with standard life testing, using over power accelerated tests, and for use in evaluating other metrics such as lumen maintenance and voltage rise. While examples of these techniques are taken from CDM lamp evaluations, the methodology is equally suited for use with other lamp types including fluorescent, induction, and solid state LED's's .

\section{Weibull distribution and historic shape factors}

In the course of life testing, conventional life estimates may be made once a portion of the population has failures. The distribution of failures might be described by several different distribution functions, but a good choice is the 2 parameter Weibull (cumulative failure) distribution given in equation (1), which is used in a variety of life and strength evaluations ${ }^{3}$.

$$
F_{t}=1-e^{-(t / \alpha)^{\beta}}
$$

When plotted in log-log form as shown in Figure 1, the shape factor, $B$, corresponds to the slope, and the scale factor, $\alpha$, corresponds to the lateral position with lifetime $(t)=\alpha$ at the 63.2 percentile. Figure 1 is an example of the Weibull distribution for life tested medium power

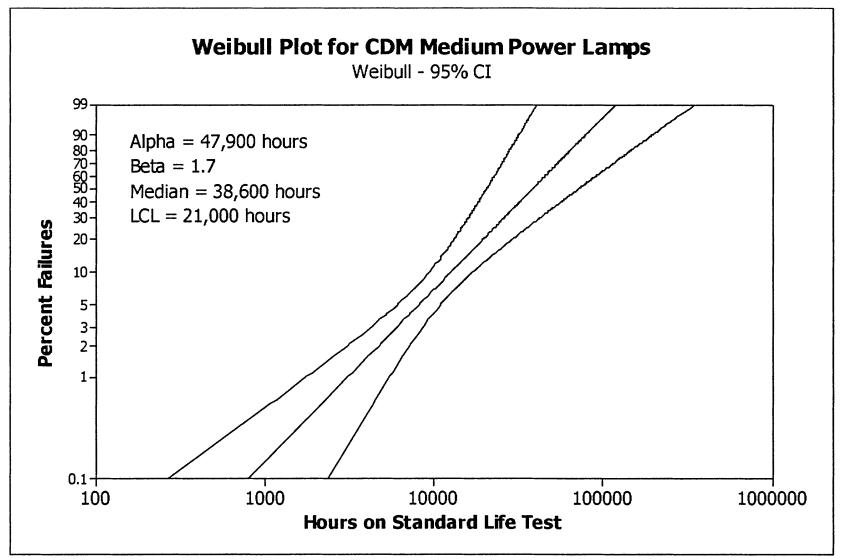

Figure 1 Weibull plot for standard life data 
CDM lamps. The left and right curves in the figure form the $95 \%$ confidence interval on the model. In this example the median life of products tested, under controlled laboratory conditions, was 38,600 hours with the $95 \%$ lower confidence level (LCL) of 21,000 hours. The actual product life, under end user conditions that are not necessarily well defined or controlled, will most likely fall within this range; the rated life of this product is 24,000 hours.

Life data are also often plotted in linear form called survival curves. Figure 2 shows examples of calculated Weibull survival curves with different shape factors but all having a 20,000 hour median life. Higher values of $B$ result from a more rapid decline in product survivals, or stated another way, a greater rate of failure than compared with products that show lower shape factor values.

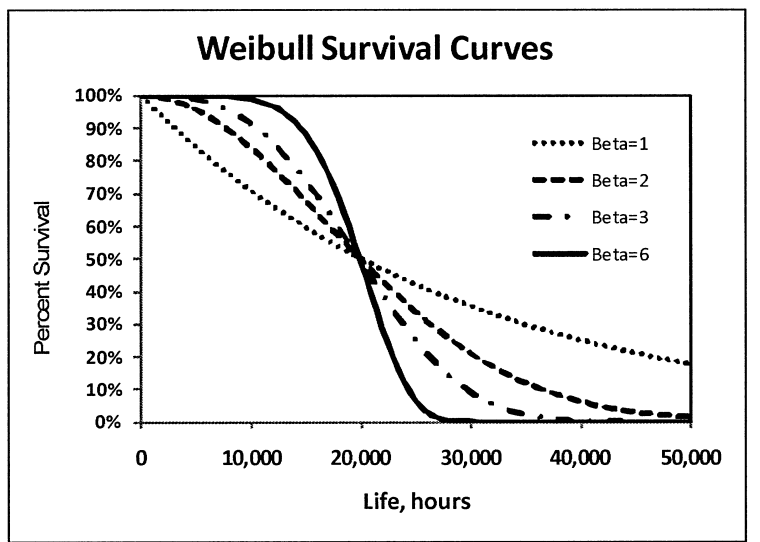

Figure 2 Weibull survival curves based on 20,000 hour median life

An analysis of five years of actual life test data was performed to establish historic life benchmark data for shape and scale factors of commercial HID lamps. Lamp life data analyzed included high-pressure sodium (HPS), quartz metal halide (QMH), and ceramic metal halide (CDM) sources. It was found that while the scale factors had a wide variety of values, which depended on the individual lamp type and rated life, the shape factors were found to be in a range of $1-3$ for all types and powers of the HID sources evaluated. Figure 3 shows the survival curves for three families of HID sources based on the historic data analysis where the shape factors are 2.25 for HPS, 1.36 for QMH, and 2.79 for CDM.

A form of reliability evaluation to project life behavior using historic data is called Bayes analysis ${ }^{4}$. By taking advantage of the historic life data, and recognizing that the majority of lamps have nearly the same shape factor and follow a Weibull distribution, we may make a statement regarding the shape factor of HID lamps: assume a shape factor of $B=3$. This is proven useful when there no, or only a few, failures present in a test, but where a more accurate estimate of the shape factor cannot be made. The choice of $b=3$ for HID lamps is made as a conservative estimate but well founded on the historic

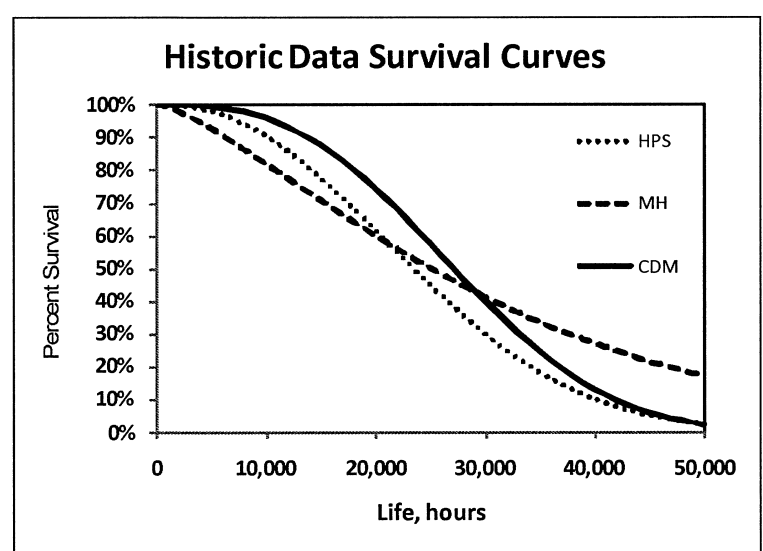

Figure 3 Weibull survival curves based on historic HID lamp life test data

data. If for example there are 30 products on test to 6000 hours (about 9 months of testing) different shape factors would give projected life times that are inconsistent with past history (and engineering experience). Figure 4 shows a Weibull plot of the calculated cumulative failure curves for this example where the shape factor is chosen as $1,2,3$, and 6 .

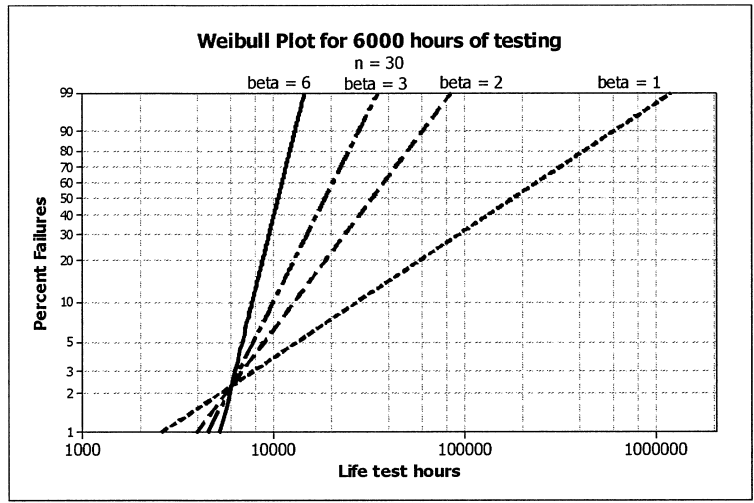

Figure 4 Weibull plot based on 6,000 hours of testing, $n=30$, and $\beta=1,2,3$, and 6

While commercial software is readily available for calculating life distributions for complex data sets that include failures and censored date, a simple approximate relationship can be found that relates the product $50 \%$ median life for a test group without failures to the number of lamps on test $(n)$, time on test $(T)$, and the historic shape factor. Starting from the Weibull distribution given in equation (1), and the ranked failure percentage ${ }^{5)}$ given in equation (2):

$$
F_{i} \approx(i-0.3) /(n+0.4)
$$


an estimate of the scale factor $\alpha$, at the test time to be evaluated $(T)$, is approximated as $\alpha \approx 0.7 n^{-1 / b}$ for large $n$ $(\geq 10)$ and the rank order at $T$ is $i=1$; a rank order of one assumes that the first failure would be imminent. This approximation of $\alpha$ allows a relatively simple equation to be derived between the special case of median product life $\left(t_{500}\right)$, number of lamps on test, test time, and the shape factor from historic data. This equation is found by substituting the above relationship for $\alpha$ into the Weibull equation (1) and solving for the median life:

$$
t_{50 \%}=n^{1 / \beta} T
$$

The utility of equation (3) can be understood for test planning purposes as well as actual life time projections. For example, 30 lamps on test without failure to 6500 hours of testing and with $B=3$, is sufficient to project a 20,000 hour median life. Therefore, this example shows that lamps need only $33 \%$ of their rated life on test to project the actual longer $50 \%$ median lifetime. The required time on test also depends on the number of lamps tested and can be used for resource planning during early stages of product development. Confidence limits can also be estimated, and in general the projected (Weibull) life at any percentile $t_{p}$ when no failures are present in the test group, is given by equation (4):

$$
t_{P}=n^{\frac{1}{\beta}} T\left[\frac{\ln \left(1-F_{P}\right)}{\ln (1-L C L)}\right]^{\frac{1}{\beta}}
$$

where $F_{p}$ is the failure percentile and $L C L$ (or $U C L$ ) is the lower (upper) required confidence level.

\section{Multi-level accelerated stress testing}

Multi-level accelerated testing is another method for reducing overall test times ${ }^{5}$. The use of multiple levels allows estimates of life under extrapolated "at-use" conditions. For accelerated testing to be useful, there is a need to have actual failures. From the failures it is possible to learn about the failure mechanisms and these can be addressed where necessary to improve product performance. The other need for failures is that life estimates are made at the stressed conditions, and these are used to extrapolate lamp life under normal operating conditions.

For accelerated stress testing to be effective at projecting lamp life at standard stress conditions, there must be two or more elevated stress levels so that extrapolations may be performed. However, it is not necessary that all lamps fail at all test levels, only enough need fail so that the $50 \%$ failure times can be found for those levels and estimates of the shape factor made; censored data is also taken into account during analysis so testing to full life even under stressed conditions is not necessary. An example plot of median life vs. relative stress levels is shown in Figure 5. It is shown how higher stress levels result in earlier median failure times that are extrapolated to the lower zero stress level to estimate the life time "at use" (zero additional stress) conditions.

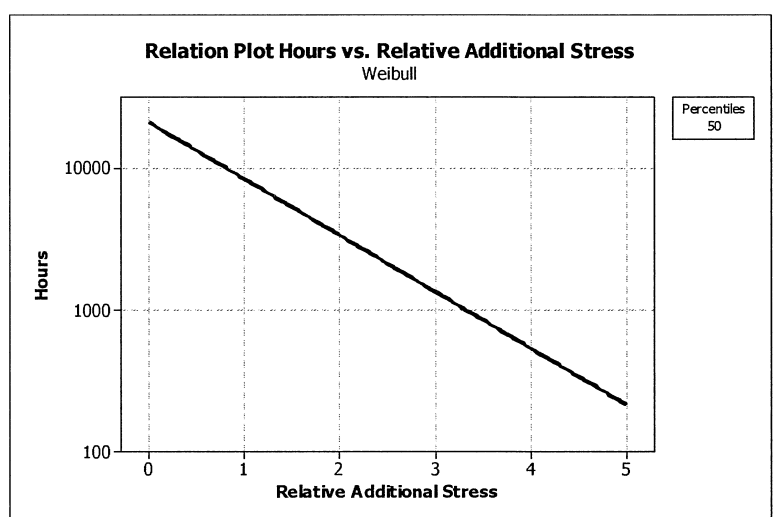

Figure 5 Relation plot between 50 percent survival and relative additional stress

A real example of stress testing medium power ceramic metal halide lamps is shown in Figure 6. Lamps were operated at $123 \%, 139 \%, 142 \%$ and $169 \%$ of rated power (powers were based on available lamp/ballast combinations). The median life for each stress level was found based on a common shape factor for all levels and estimated from the actual failures times. In case of no failures at a particular level the data are all censored but a median life is still calculated following the discussion in section 2. These data are plotted on a log - linear plot shown in Figure 6 . The projected median life from this test is 29,600 hours. The shape factor of 2.4 is based on the actual failure data and applied to all levels in this analysis.

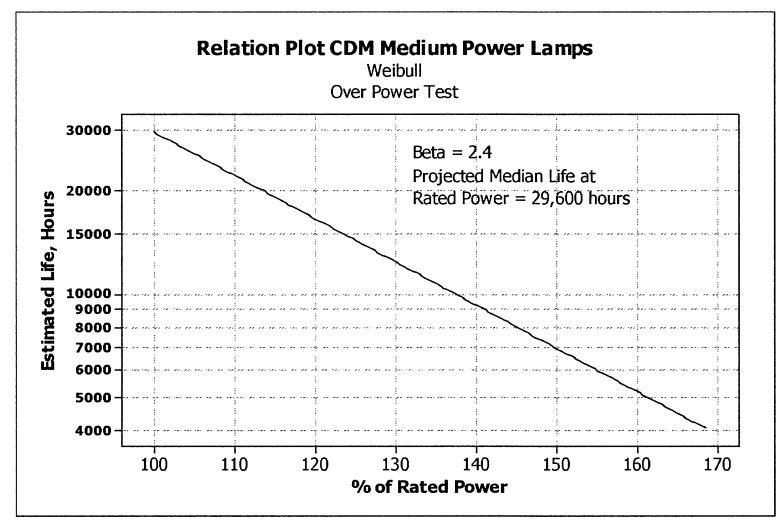

Figure 6 Relation plot for CDM lamps on over power test

A linear regression model is obtained from these analyses in the form:

$$
\ln \left(\alpha_{\text {stress }}\right)=C_{1} \times \text { Stress }+C_{2}
$$


From this model, acceleration factors, $A F$, may be calculated:

$$
A F=\alpha_{\mathrm{o}} / \alpha_{\text {stress }}
$$

Where $\alpha_{o}$ is the Weibull scale factor at use, conditions; $a_{\text {stress }}$ is the scale factor at the stressed level; $C_{1}$ and $C_{2}$ are constants based on the model; $C_{1}=-0.03$ and $C_{2}=13.3$ in this example. Other regression stress models may also be appropriate; the chosen model can be selected reliably if the background of the failure cause is known. A plot of the acceleration factor vs. percent of rated power for this over power test is shown in Figure 7. It can be observed that modest increases in lamp power can yield a significant reduction in test time until failure. For example $50 \%$ over power may result in $1 / 4$ of the testing time. Care must be exercised not to force failures at too high stress levels that result in failures inconsistent with actual practice. The real utility of multi-level stress testing is that standard reliability analysis techniques allows for the use of censored as well as failed data, so that even the accelerated tests need not be run to the end of life and life projections can be made relatively early during performance evaluations. This is especially valuable when failures occur earlier than expected based on previously established $A F$ values indicating early on that there may be trouble with a pending design. However, acceleration factors may be different for different products so proper engineering practice should be exercised.

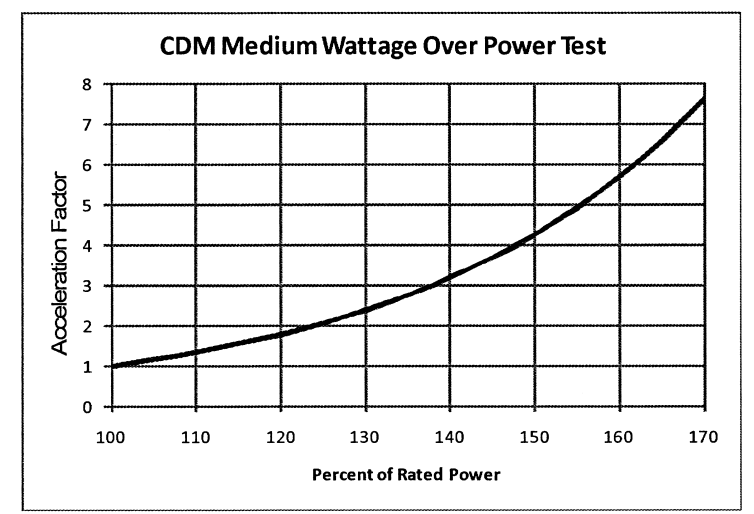

Figure 7 Acceleration factor as a function of over power

Operating power is but one example of an external stressor and is not the only stress available to the lamp developer; there are other external and internal stressors that may be utilized to speed product development and establish overall reliability. Other external stressors can be rapid on/off cycling, temperature cycling, vibration testing, etc. Internal stressors are those related to design aspects such as electrode size, chemistry variations, arc tube wall loading, and so forth. The use of internal and external stressors, individually, or in combination, can lead to very powerful reliability evaluation tools.

Over power and other accelerated or stress testing may also uncover potential troublesome failure mechanisms that are not likely to be seen in early life evaluations. This can be useful in products that have rated lifetimes that can reach tens of thousands of hours. Additionally, there can be multiple failure mechanisms, and these can have separate acceleration factors as well. Properly designed stress tests and observant failure analysis can distinguish between different failure mechanisms, and these are handled through data failure mode censoring. Commercial reliability software makes these types of analysis fairly straight forward. There is also a class of early failures referred to as catastrophic or manufacturing defect failures. While this is an important class of failure, especially with regard to product warranty, they can influence product life projections. However, when analyzed and given an assignable cause not related to normal product wear, these types of data can be censored from the test population if the analysis warrants. Further discussion of failure mechanisms, however, is beyond the scope of the present work.

\section{Virtual failure analysis}

The same techniques used in standard and accelerated testing to establish operational life, can also be used on other indicators such as color shift, lumen maintenance, voltage rise, and other performance metrics that change or degrade over life. This is done by defining virtual failures as those that do not meet an arbitrary performance standard. Failure statistics are then applied to the population. Analysis of the virtual failures in the context of an accelerated test can then be used to predict or confirm the actual performance at standard use conditions for that performance indicator. A further advantage is that the use of virtual failures yields an analogue response instead of a digital yes/no failure, improving the statistical analysis.

A common performance metric in lamps is voltage rise. One form of actual failure may be when the lamp voltage exceeds the available ballast sustaining voltage (magnetic systems) or the bus voltage (electronic drivers). In one example, the virtual failure time was arbitrarily defined as the time after which the voltage rise was greater than 5 volts. Lamps operating over power were used in the analysis. The results, shown in Figure 8, project that at rated power a 5 volt rise can be expected, on average, after 5700 hours of operation. Standard life data shows that the average voltage rise of 5 volts occurred at 5500 hours, in close agreement.

Another example of virtual failure analysis is with lumen maintenance in medium power CDM lamps. In an over power test, the virtual failure point is (arbitrarily) 


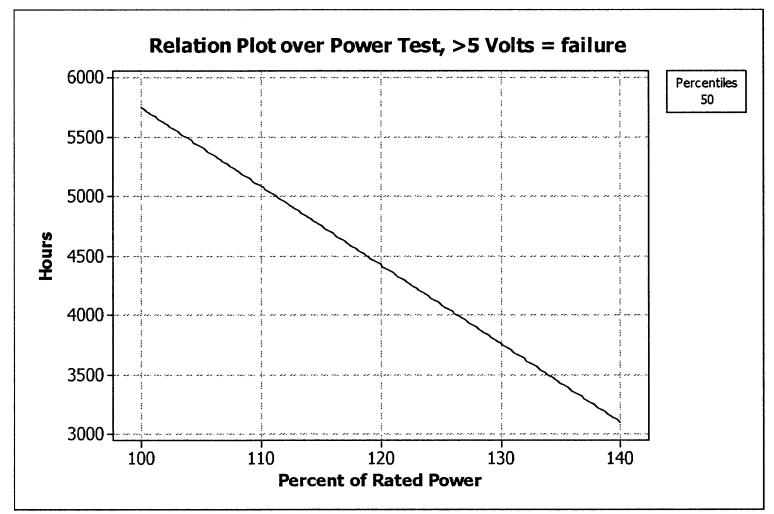

Figure 8 Relation plot for voltage rise vs. percent of rated power

defined as $\mathbf{9 0 \%}$ lumen maintenance. Figure $\mathbf{9}$ shows the experimental results. The time to reach $90 \%$ lumen maintenance was projected to be 12,600 hours. Under

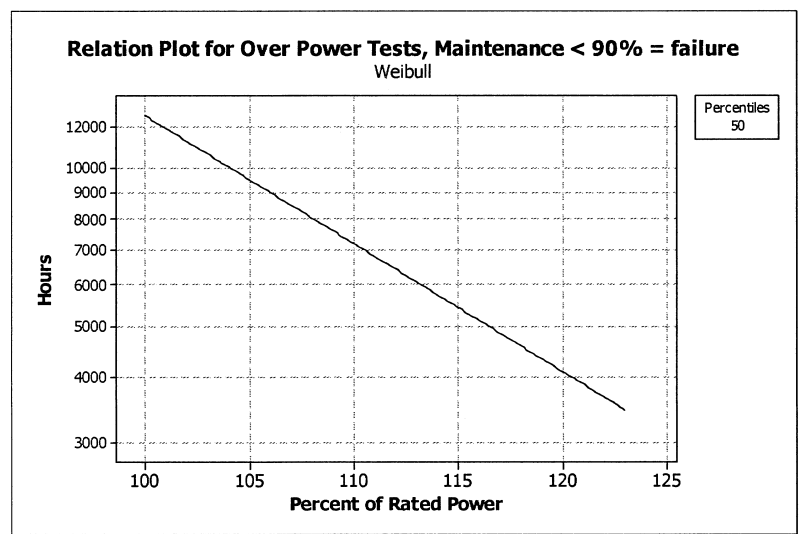

Figure 9 Plot of time to reach $90 \%$ lumen maintenance vs. over power level

normal life test conditions, the actual time was found to be 11,500 hours in close agreement.

One of several uses of this type of analysis is for early evaluation of product performance (other than life). In actual practice, a product is not as likely to be operated in the controlled environment of the testing laboratory and therefore this type of analysis may be helpful in understanding the risk in stressed conditions and establishing performance de-rating data. Another use can be when evaluating known failure modes such as chemical corrosion of wall materials or erosion of electrodes in HID lamps ${ }^{6}$. With X-ray techniques, erosion vs. tube wall thickness can be used as a metric with a percentage of corrosion acting as the virtual failure point thereby anticipating end of life prior to any actual failures.

\section{Conclusions}

Predicting operational life in long life lamps, such as ceramic metal halide lamps, can be accomplished in significantly shorter periods than waiting for $50 \%$ of the products to actually fail by utilizing reliability techniques. Especially useful is the Weibull distribution and the use of a historic based shape factor when no failures have occurred in the early stages of testing. This information can be augmented through the use of multilevel accelerated stress testing where extrapolation to at-use conditions compares favorably to standard life testing when run using multiple stress levels. The use of virtual failures in both standard and accelerated testing can further enhance the estimation of product performance over life, well in advance of the tens of thousands of hours of actual lamp operation. These methodologies find use in many other areas of lighting and are well suited not only for HID source development, but in the evaluation of lamp and ballast systems, and especially with the reliability of extreme long life products such as LEDs and electrode-less induction lighting sources.

\section{References}

(1) Philips on line catalog, MasterColor CDM-T MW Elite $315 \mathrm{~W} / 930 \mathrm{http}: / / \mathrm{www}$.ecat.lighting.philips.com

(2) Evertz, Driel, W.D van, Kloosterman, J., Vanlier, G., and Zhang G.Q.: Towards a System Level Reliability approach for Solid State Lighting, Proc. 12th Inte. Symp. Sci. Technol. Of Light sopurces LS12-WLED p.225 (2010).

(3) Tóth, Z., Juhász, A. and Nyíri, B.: Evaluation of the Mechanical Properties of HID Sealing during Ageing, Proc. 9th Inte. Symp. Sci. Technol. of Light Sources LS9, p. 381 (2001).

(4) Singpurwalla, N.: Reliability and Risk; A Bayesian Perspective, John Wiley \& Sons, N.Y. (2006).

(5) Nelson, W.: Accelerated Testing; Statistical Models, Test Plans, and Data Analyses, John Wiley \& Sons, N.Y. (1990).

(6) Methling, R., Fanke, S., Schöpp, H. and Kaening, M: X-Ray Computer Tomography in End-of-Life Investigations of HID Lamps, LEUKOS Vol.7, No.4, April 2011, pp. 237-239 (2011). 